\title{
Real-Time Face Recognition by the PCA (Principal Component Analysis) with Color Images
}

\author{
Jin Ok Kim ${ }^{1}$, Sung Jin $\mathrm{Seo}^{2}$, and Chin Hyun Chung ${ }^{2}$ \\ 1 Faculty of Multimedia, Daegu Haany University, \\ 290, Yugok-dong,Gyeongsan-si, Gyeongsangbuk-do, 712-715, KOREA \\ bit@dhu.ac.kr \\ 2 Department of Information and Control Engineering, Kwangwoon University, \\ 447-1, Wolgye-dong, Nowon-gu, Seoul, 139-701, KOREA \\ chung@kw.ac.kr
}

\begin{abstract}
The face recognition by a CCD camera has the merit of being linked with other recognition systems such as an iris recognition to implement a multimodal recognition system. This paper is concerned with a new approach to face recognition that is automatically distinguished from moving pictures. Based on the research about recognition of color image by a CCD camera, we first find the proper value of color images in order to distinguish the tone of skin from other parts of face. Then, we look for the skin color among the regions of skin color converting RGB into $Y, C b, C r$ to find skin parts of face. This new method can be applied to real-time biometric systems. We have developed the approach to face recognition with eigenface, focusing on the effects of eigenface to represent human face under several environment conditions.
\end{abstract}

\section{Introduction}

Face recognition is used to identify one or more persons from still images or a video image sequence of a scene by comparing input images with faces stored in a database. The application of face recognition technology can be categorized into two main parts: law enforcement application and commercial application. Face recognition technology is primarily used in law enforcement applications, especially mug shot albums and video surveillance. The commercial applications range over static matching of photographs on credit cards, ATM cards, passports, driver's licenses, and photo IDs to real-time matching with still images or video image sequences for access control. Each presents different constraints in terms of processing requirement.

This paper presents new technology that recognizes a face and automatically distinguishes it from moving pictures. Since difficult problems in recognizing color image by a CCD camera were handled in the prior research to make face region less sensitive [1. We find out the proper value of color images in order to distinguish the tone of skin from other parts of face 2]. We look for the skin color among the regions of skin color converting RGB into $\mathrm{Y}, \mathrm{Cb}, \mathrm{Cr}$ and define the skin color by using $Y, C b, C r$ to find skin parts [3] 4]. 


\section{Face Detection and Face Recognition}

In this section, we present a process of implementing face recognition and posing important factors. Lighting and angle of a pose have an effect on face detection. Particularly, it is hard to find the face color and face shape by an effect of lighting [5] 6]. Since most significant elements in facial detection can be changed by the angle of face, eyes and mouth, we should define the face's angle to obtain some necessary data within the significant elements.

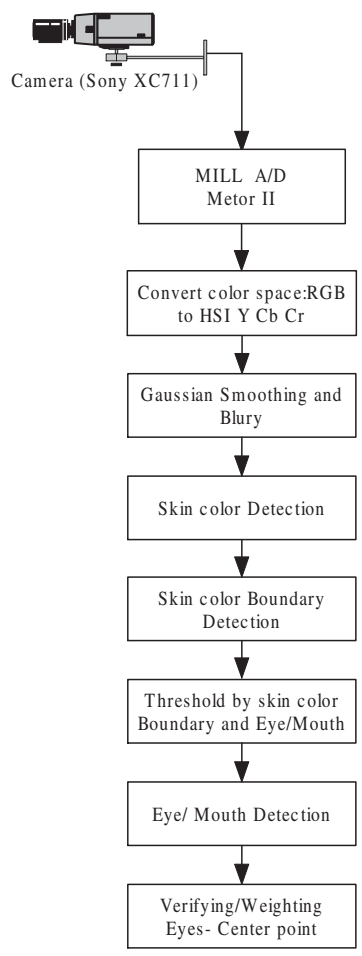

Fig. 1. Face detection algorithm

The proper value of color images is needed to distinguish the tone of skin from other parts of face [2] [7. Moreover, we make sure that detection of the necessary facial part is done by using one of masks among $3 \times 3,5 \times 5$ or dynamic size. The detection of the characteristic color value is done with Gaussian distribution. After the face is detected, the rectangle area is extracted.

Color space taken by a CCD camera should be converted from RGB to HSI and $\mathrm{Y}, \mathrm{Cb}, \mathrm{Cr}$ to get specific ranges of $\mathrm{H}, \mathrm{S}, \mathrm{Cb}$ and $\mathrm{Cr}$ [8].

Figure 2 is about procedure of skin color detection. 


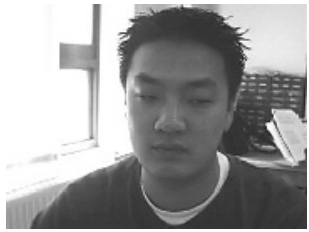

(a) Original image

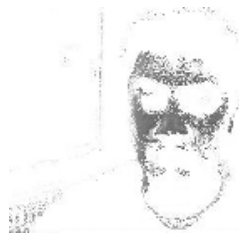

(b) Skin color image

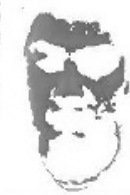

(c) Processed color image

Fig. 2. Detection of skin regions
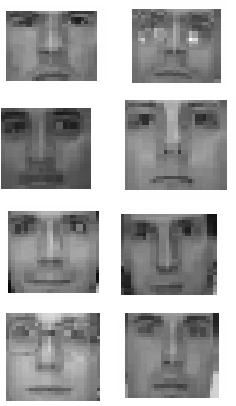

(a) From face image set
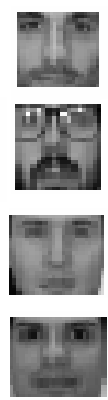
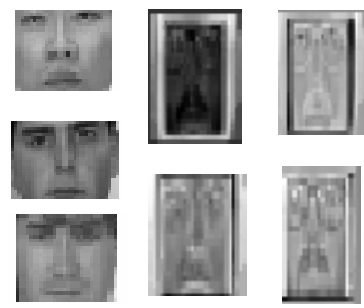

(b) From eigenface
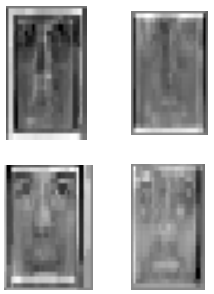

(c) After test image

Fig. 3. Face detection

\section{Experimental Results}

The region of skin color on face can be detected by experiments. Specially, we tried to search out the face, eyes and mouth by using division of $\mathrm{Cb}, \mathrm{Y}, \mathrm{Cr}$ and RGB of skin color. Through the binarization, we found out the significant elements from the face region.

Since the face could be calculated and displayed numerically, we could distinguish the face. With definition of the region of width or center value of the eyes, we could distinguish the data when a face shows front pose.

Based on the result that error rate of detection decreases and recognition rates increases, we could process the real-time recognition with the data.

We carried out the experiment for the facial recognition with 16 images. We defined the eigenface as eight eigenvectors from each corresponding eigenvalue. 
To decrease the rate of error, we use front facial region that includes the significant elements tested in Section 2. Fig. 3(b) shows the eigenface and Fig. $3(\mathrm{c})$ shows that we process the facial recognition from the test images

\section{Conclusion}

We proposed a face detection algorithm for color image captured by a CCD camera by using a skin-tone color model and features. Using the distance and width of significant elements like eyes and mouth, we could easily detect whether an object on monitor is an human or a picture. If face information captured several times is compared to the images in DB, we could recognize who he/she is. So, we could decrease the recognition error rate. Our method first corrects the color that automatically estimates the white reference pixel. We search $Y$, $\mathrm{Cb}, \mathrm{Cr}$ skin color space. Our method detects skin region over the entire image and then generates face candidates based on the spatial arrangement of the skin patch. Our algorithm reconstructs eyes, mouth, and boundary map to verify the face candidates. And we designed the face recognition system through the PCA. The system shows the exact recognition results for the 16 persons. However, we found some errors after changes to the different poses and backgrounds. To decrease this errors, we use the DB of the captured images. Finally, we could decrease the errors and make the system stable.

Acknowledgements. The present Research has been conducted by the Research Grant of Kwangwoon University in 2004.

\section{References}

1. Hjelmas, E., Low, B.K.: Face detection: A survey. Computer Vision and Image Understanding 83 (2001) 236-274

2. Yang, J., Waibel, A.: A real-time face tracker. Proc. Third Workshop Application of Computer Vision (1996) 142-147

3. Donato, G., Bartlett, M.S., Hager, J.C., Ekman, P., Sejnowski, T.J.: Classfiying facial actions. IEEE Transactions on Pattern Analysis and Machine Intelligence 21 (2000) 974-989

4. Kjeldsen, R., Kender, J.: Finding skin and guesture recognition. In: Proc. 2nd Int'l Conf. on Automatic Face and Gesture Recognition. (1996) 312-317

5. Yang, M., Kriegman, D.J., Ahuja, N.: Detecting faces in images: A survey. IEEE Transactions on Pattern Analysis and Machine Intelligence 24 (2002) 34-58

6. Crow, I., Tock, Bennett, A.: Finding face features. In: Proc.Second European Conf., Computer Vision (1992) 92-96

7. Mckenna, S., Gong, S., Raja, Y.: Modelling facial colour and indentily with gaussian mixtures. Pattern Recognition 31 (1998) 1883-1892

8. Wang, Y., Osterman, J., Zhang, Y.Q.: Video processing and communications. Probability and Random Processes with applications to Signal Processing (2002) 24-25 